\title{
Tratamento de aneurisma de aorta justarrenal com stent modulador de fluxo
}

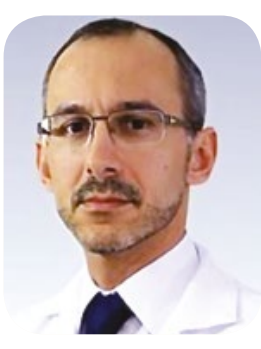

\section{INTRODUÇ̃̃O}

O tratamento dos aneurismas de aorta abdominal (AAA) justarrenais ou toracoabdominais é um desafio para o cirurgião vascular, uma vez que há acometimento de ramos viscerais (tronco celíaco, artéria mesentérica superior, artérias renais e ramos medulares) ${ }^{1}$. A cirurgia aberta para correção desses aneurismas ainda apresenta alta morbimortalidade, mesmo em centros de referência ${ }^{2}$. Na busca de melhores resultados, outras técnicas foram desenvolvidas, como cirurgias híbridas (combinação do tratamento endovascular combypass visceral), implante de endopróteses fenestradas ou ramificadas, implante de endopróteses com "stents em paralelo" e osstents moduladores de fluxo ${ }^{1,3,4}$.

Apresentamos um paciente portador de AAA sacular justarrenal de grande diâmetro - elevada probabilidade de rotura -, com alto risco cirúrgico, em decorrência de, entre outros fatores, recente infarto do miocárdio. Diante do quadro clínico complexo, fez-se necessário o tratamento do aneurisma de forma pouco invasiva, reduzindo o tempo cirúrgico e volume de contraste, o que foi possível com implante destent modulador de fluxo.

\section{CASO CLÍNICO}

Paciente de 84 anos, masculino, hipertenso e coronariopata, realizou ultrassom abdominal de rotina com diagnóstico incidental de AAA. Submetido à angiotomografia, evidenciou uma dilatação sacular na aorta abdominal justarrenal com $8,0 \mathrm{~cm}$ de diâmetro e outra dilatação fusiforme na aorta distal medindo $5,5 \mathrm{~cm}$ de diâmetro (Foto 1).

Indicada intervenção devido ao risco de rotura, por tratar-se de aneurisma sacular de grande diâmetro; dentre as possibilidades terapêuticas, optou-se pelo tratamento endovascular, o que seria possível com implante de endoprótese fenestrada/ramificada, técnica de sanduíche/snorkel ou uso de stent modulador de fluxo.

Durante avaliação e preparo pré-operatório, evoluiu com infarto agudo do miocárdio e foi submetido à an-

\author{
Maurício de Amorim Aquino ${ }^{1}$
}

gioplastia de urgência, com uso de stent farmacológico, mantendo-se posteriormente em dupla antiagregação. Após 45 dias, foi submetido ao tratamento endovascular com escolha pelo implante de stent modulador de fluxo, por tratar-se de procedimento de menor morbimortalidade, com menor tempo cirúrgico e menor volume de contraste.

O procedimento foi realizado na sala de hemodinâmica, sob anestesia geral, através de punção da artéria femoral comum direita com passagem de cateter pigtail para controle angiográfico e dissecção da artéria axilar esquerda para passagem do stent modulador de fluxo Multilayer(MFM ->) $30 \times 180 \mathrm{~mm}$, o qual foi posicionado cobrindo da aorta torácica descendente até a aorta terminal (bifurcação das ilíacas). O paciente foi extubado imediatamente após o procedimento, permanecendo 24 horas em Unidade de Terapia Intensiva. Obteve alta hospitalar no segundo dia pós-operatório. Em nenhum momento foi suspensa a dupla antiagregação, o que aumentaria o risco de novo evento coronariano. O Duplex Scan de controle, realizado 3 meses depois, e as angiotomografias com 6 e 18 meses após o procedimento (Foto 2), mostraram o saco aneurismático excluso com perviedade de todos os ramos viscerais.

\section{DISCUSSÃO}

Os AAA justarrenais ou envolvendo artérias viscerais são classificados como aneurismas toracoabdominais, uma vez que a abordagem cirúrgica aberta geralmente necessita de toracofrenolaparotomia para sua adequada exposição e correção5,6. Embora a cirurgia convencional seja efetiva e com baixas taxas de reintervenção a longo prazo, a mesma apresenta taxas de morbimortalidade elevadas, principalmente em pacientes com alto risco operatório, pelo seu caráter mais invasivo necessidade de dissecção cirúrgica extensa com clampeamento da aorta torácica e maior risco de isquemia visceral e paraplegiai ${ }^{1,2,4}$.

A evolução da técnica endovascular, com uso de endopróteses de menor perfil, permitindo em alguns casos 
o implante totalmente percutâneo, tornou esse método a primeira opção para o tratamento dos aneurismas da aorta $^{5}$. Para a exclusão adequada do aneurisma e selamento dos pontos de ancoragem das endopróteses, a presença de ramos viscerais é um fator complicador. Por se tratarem de próteses impermeáveis, a cobertura desses ramos promoveria isquemia dos órgãos envolvidos. Assim, houve a necessidade do aperfeiçoamento da técnica com uso associado de bypass (cirurgia híbrida), uso de stents em paralelo (técnica do snorkel ou sanduíche) e do desenvolvimento de próteses ramificadas ou fenestradas ${ }^{1,3,4}$. Entretanto, todas essas técnicas ainda têm resultados limitados, pelo risco de oclusão de ramos ou vazamentos entre as conexões das próteses.

Nos últimos anos, o stent modulador de fluxo- Multilayer (MFM ->) - surgiu como uma nova estratégia terapêutica para os aneurismas toracoabdominais: próteses metálicas com múltiplas camadas, não revestidas por tecido, que, mesmo sem provocar a exclusão completa do fluxo no saco aneurismático, reduzem a pressão no seu interior, impedindo a rotura. Esses materiais promovem não uma barreira mecânica, mas hemodinâmica, remodelando o fluxo no aneurisma - o fluxo passa de turbilhonado para laminar - com consequente trombose gradual em camadas, porém preservando o fluxo sanguíneo em seus ramos ${ }^{7,8}$.

Uma recente metanálise mostrou que, quando aplicado dentro das instruções de uso (IFUs), o tratamento com implante do Multilayer (MFM $\rightarrow$ ) apresentou baixa taxa de mortalidade em 30 dias (2,9\%) e sobrevida de $93,3 \%$ em 18 meses $^{4}$. Não houve nenhum caso de paraplegia, infarto ou necessidade de hemodiálise. Os ramos viscerais envolvidos nos aneurismas e cobertos pela malha do stent apresentaram uma taxa de perviedade de $97,8 \%$. Deve-se ressaltar que todos os pacientes tratados eram de alto risco cirúrgico (ASA $\geq$ III), que apresentavam contraindicação clínica ou anatômica para outros métodos terapêuticos (cirurgia aberta ou mesmo endovascular, como uso de próteses ramificadas etc).

Ainda são necessários mais estudos de acompanhamento a longo prazo para definir a possibilidade da ampliação do uso do Multilayer (MFM- $>$ ) como primeira escolha na correção dos aneurismas da aorta. Apesar das vantagens que apresenta no tratamento de aneurismas com envolvimento de ramos arteriais importantes, deve-se lembrar que a técnica também apresenta limitações - como no caso de aneurismas rotos, aneurismas micóticos, vasculites, entre outros. Os casos devem ser bem selecionados, seguindo rigorosamente as instruções de uso recomendadas pelo fabricante. Os pacientes tam- bém necessitam de dupla antiagregação por período mínimo de três meses após o procedimento, pelo risco de oclusão dos ramos cobertos pelo stent.

No caso relatado, diante das comorbidades apresentadas pelo paciente, as quais aumentavam substancialmente o risco operatório por qualquer outra técnica, o tratamento com Multilayer (MFM- $>$ ) apresentou resultados que superaram as expectativas a curto prazo e tem se mantido satisfatório com 18 meses de seguimento, como demonstrado nos exames de acompanhamento por imagem tomográfica. O método, porém, não pode ser visto como solução para todas as doenças complexas da aorta e os critérios técnicos de indicação e implante devem ser criteriosamente respeitados para o sucesso do tratamento.

\section{FIGURAS}

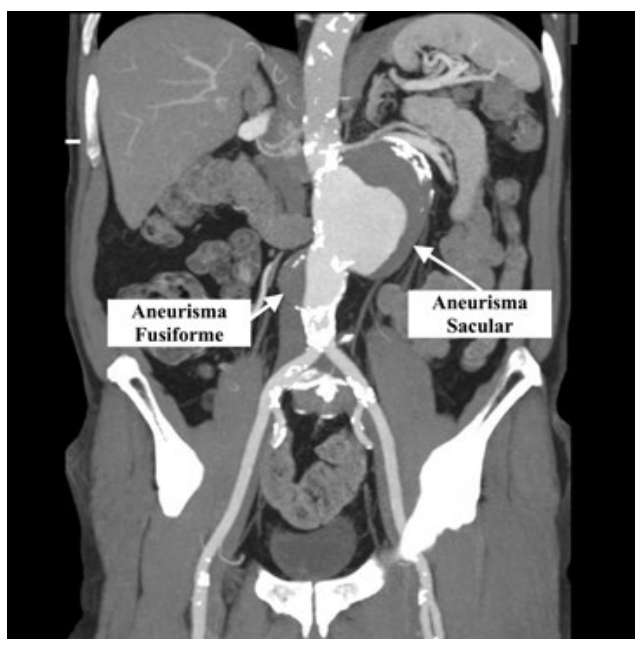

Figura 1 - Angiotomografia pré-operatória mostrando aneurisma sacular justarrenal proximal e outro fusiforme na aorta distal.

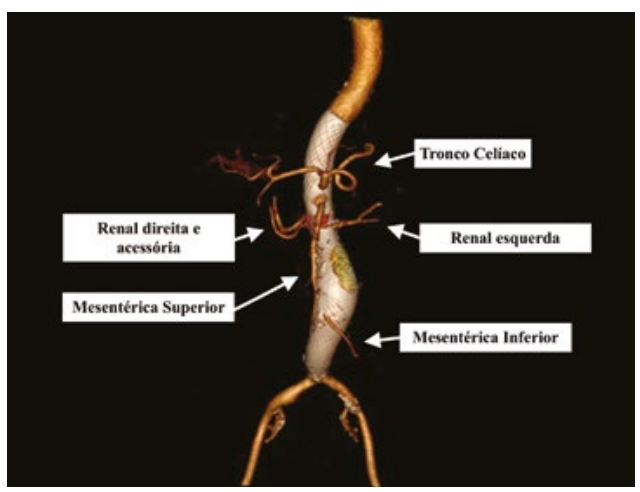

Figura 2 - Angiotomografia pós-operatória mostrando exclusão dos aneurismas, com todos os ramos viscerais pérvios. 


\section{REFERÊNCIAS}

1. Lobato AC, Camacho-Lobato L. Endovascular treatment of complex aorticaneurysms using the sandwich technique. J EndovascTher. 2012 Dec;19(6):691-706.

2. JCS Joint Working Group. Guidelines for diagnosis and treatment of aortic aneurysm and aortic dissection (JCS 2011): digest version. Circ J. 2013;77(3):789-828.

3. Fillinger MF, Greenberg RK, McKinsey JF, et al; Society for Vascular Surgery Ad Hoc Committee on TEVAR Reporting Standards. Reporting standards for thoracic endovascular aortic repair (TEVAR). J Vasc Surg. 2010 Oct;52(4):1022-33, 1033.e15.

4. Hynes N, Sultan S, Elhelali A, et al. Systematic Review and Patient-Level Meta-analysis of the Streamliner Multilayer Flow Modulator in the Management of Complex Thoracoabdominal Aortic Pathology. J EndovascTher. 2016 Jun;23(3):501-12.

5. Erbel R, Aboyans V, Boileau C, et al; ESC Committee for Practice Guidelines. 2014 ESC Guidelines on the diagnosis and treatment of aortic diseases: Document covering acute and chronic aortic diseases of the thoracic and abdominal aorta of the adult. The Task Force for the Diagnosis and Treatment of Aortic Diseases of the European Society of Cardiology (ESC). Eur Heart J. 2014 Nov 1;35(41):2873-926. doi:10.1093/eurheartj/ ehu281. Epub 2014 Aug 29. Erratum in: Eur Heart J. 2015 Nov 1;36(41):2779.

6. Jaldin RG, Sobreira ML, Moura R, et al. Tratamento de aneurisma de aorta sacular justarrenal com Multilayer Flow Modulator: relato do primeiro caso realizado em Hospital Público no Brasil. J. vasc. bras. 2014 Set;13(3): 235-243.

7. Vaislic CD, Fabiani JN, Chocron S, et al; STRATO Investigators Group. One-year outcomes following repair of thoracoabdominal aneurysms with the multilayer flow modulator: report from the STRATO trial. J EndovascTher. $2014 \mathrm{Feb} ; 21(1)$ : 85-95.

8. Oliveira JR, Aquino Mde A, Barros S, et al. Alterations of blood flow pattern after triple stent endovascular treatment of saccular abdominal aortic aneurysm: a porcine model. Rev Col Bras Cir. 2016 May-Jun;43(3):154-9.

1- Serviço de Angiologia e Cirurgia Vascular do HSI Endereço para correspondência:

aquino.ma@gmail.com 